UNITED STATES DEPARTMENT OF THE INTERIOR

GEOLOGICAL SURVEY

\title{
Principal facts for a gravity survey of the Ennis, Montana Geothermal Area
}

by

R. M. Senterfit

U. S. Geological Survey

Box 25046, Denver Federal Center

Denver, Colorado 80225

Open-file Report 80-98

1980 
On November 4, 1978, thirty-three gravity stations were established about one mile north of Ennis, Montana, in the Ennis geothermal area, southwestern Montana (fig. 1).

Elevations for the stations were determined by transit level ( $R$. Leonard, U.S. Geological Survey, written commun., 1978) and by benchmark or spot elevations shown on the U.S. Geological Survey topographic map of Ennis at a scale of $1: 62,500$.

The gravity readings were made with a LaCoste-Romberg ${ }^{1}$ gravimeter (number g-235) having a scale factor of about one milligal per division.

A base station was occupied at the beginning and end of the day of metering (fig. 1). The observed gravity was referenced to the U.S. Department of Defense base station (ACIC 0475-0) in Helena, Montana (fig. 2), having a value based on the International Gravity Standardization Net, 1971 (Defense Mapping Agency Aerospace Center, 1974).

The Geodetic Reference System 1967 formula (International Association of Geodesy, 1967) was used to compute theoretical gravity.

The two Bouguer anomalies were computed by use of the following U.S.G.S. computer programs:

1.) U.S.G.S. Gravity Reduction System (R. H. Godson, D. Dansereau, and R. Sweeney, unpub. data, 1978)

2.) Program Bouguer (R. H. Godson, U.S.G.S. unpub. data, 1978)

${ }^{1}$ Use of brand names in this report is for descriptive purposes only, and in no way consitutes endorsement by the U.S. Geological Survey. 
Terrain, tidal, and drift corrections were made with the above two programs. All corrections were made from each station to a distance of $167 \mathrm{kilometers.} \mathrm{Densities} \mathrm{of} 2.67 \mathrm{~g} / \mathrm{cm}^{3}$ and $2.50 \mathrm{~g} / \mathrm{cm}^{3}$ were used in computing the Bouguer anomaly. 


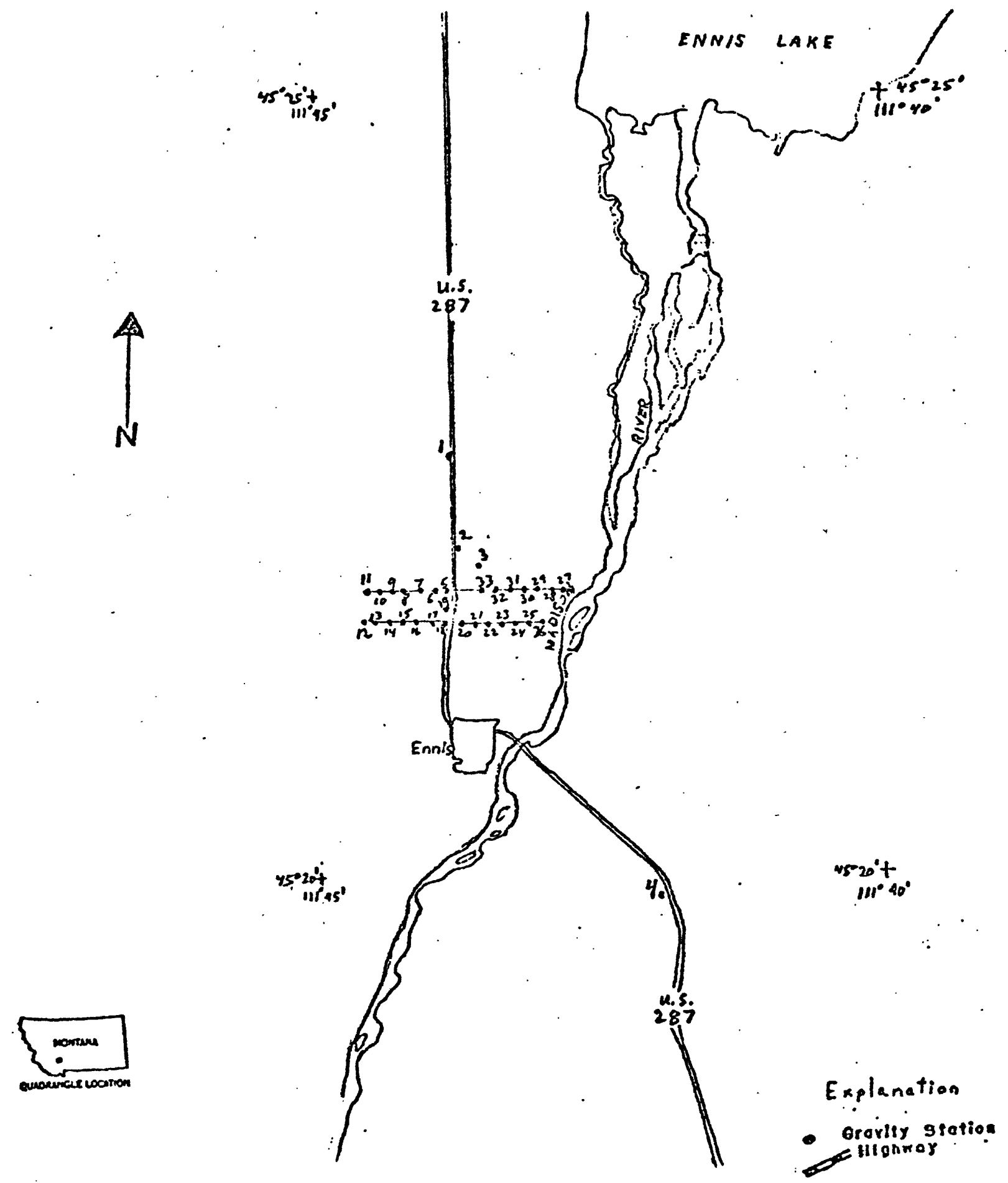

Fig.1 GRAVITY STATION LOCATION MAP, ENNIS GEOTHERMAL AREA;

ENNIS . MONTANA

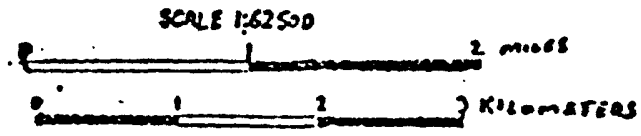


Explanation of the headings of the accompanying table of principal facts:

STATION IDENT IFICATION

LATITUDE

LONGITUDE

ELE

ST

OBSERVED

THEORETICAL

TERRAIN

BOUGUER

CURV

FREE AIR

COMPLETE-BOUGUER
Gravity station number.

North latitude in degrees, minutes

and hundredths of minutes.

West longitude in degrees, minutes

and hundredths of minutes.

Elevation in feet (to convert to

meters, multiply by 0.3048 ).

State identification (Montana).

Observed gravity in milligals.

Theoretical gravity in milligals.

Terrain correction in milligals.

Bouguer correction in milligals.

Curvature correction in milligals.

Free-air anomaly in milligals.

Bouguer anomaly in milligals, based on densities of 2.67 and 2.50 . 
$\infty \stackrel{0}{2}$

$000 \pi$

แ

$\infty \frac{0}{0}$

10

$-\omega$

$x$ a 11

$a$ 王

2

$-w_{\alpha} \alpha$

1

$\infty w$

$2 \frac{a}{c 0}$

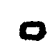

$-\frac{2}{4}$

w

$\propto 3$

$\simeq$ ?

$c$

$2=$

$\frac{a}{x}$

2

H

$\infty$

ธับ

3

$-1$

(1) $\because \div \because \div 3$

$m-=n n$ D o $0 \mathrm{~m}$

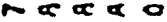
$\overrightarrow{1} \overrightarrow{1}=2$

nnm

no. $0 \mathrm{mn}$

ติำ용

ค.า.?

응ㅇㅇ을

000

0000

궁웡영

$\div \overrightarrow{1} \div \overrightarrow{1}$

cm $=00$

중

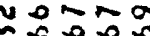

$\because \div 1 \frac{1}{11}$

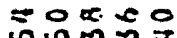

$\therefore \div-\div$

w

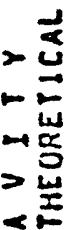

ำำ

$\because \dot{i} i^{\circ}$

৩心シ

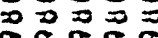

$\infty 0$

$\rightarrow \frac{1}{4}$

药

모

웅중

imio

드쇼

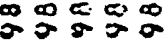

0

Node moin

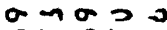
ODM $=0$

$\infty$

$2 \omega$

il

$c^{m}=$

isc $\overrightarrow{0} \approx \overrightarrow{0} \approx \overrightarrow{0}$

- 을

$<$ 년:

$\omega$

O

号

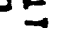

$\pm 0$

$\div \div$

in in

$2 x \stackrel{n}{n}$

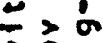

20

$\leqslant 00$

2

$1 \div 2$

$-\approx$

$5 \div \div$

in in i $0<50$ $\overrightarrow{1} \overrightarrow{1} \overrightarrow{1}$

NRmnc $\infty$ in o o $1 \% 1 \%$

Oำก

$\infty$ nm n $5 n=0$

$\div \div \div \div$

윽ㅇㅇ응

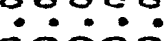

00000

영영당

$\because \ddot{1} \div 13$

- $x$ vin

ㅇ?ㄴ?

$\infty 200$

$\div \div \div \overline{1}$

$5 \cong \tilde{5}$

$m=\pi \Omega$

25535

ЕЁ Ё Е

00000

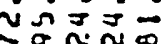

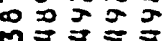

in $=5$

$\ddot{Z}=ニ=$

$\div \overrightarrow{1}: \overrightarrow{1}$

둥ㄷㅇ

$\dot{m} \dot{\sim} \dot{0}$

制ñ

c⿻n𠃋? $=$

55053 inisis

.

$\bar{E} \Xi \tilde{E}$

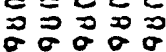

$\because \approx D ⿻ 0$

$m=\therefore<$

프는

$55 \overline{5} 5$

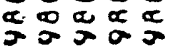

Eै E E E E

00000

$\dot{0} \dot{0}=$

nNNNN

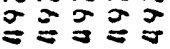

으을

$\propto 0-n=$

mं $=\dot{3}=$

$-0=-\infty$

$\div \div \div=\square$

num nn

ot 0 D

nunun

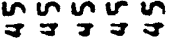

moroo

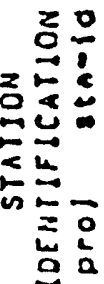

- - Nm

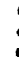

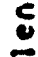
$\infty \infty \propto N$

$\because \dot{0} \dot{0}$

$\therefore \dot{0} \dot{0} \dot{0}$

$\therefore \div$ $\therefore 0000$

$\div \frac{1}{1} \div$

cenos

orán

$\vec{a} \overrightarrow{1}$

rom $\rightarrow N$

$\therefore \therefore \therefore \therefore$

$\div \div \div$

$\vec{n} \boldsymbol{m} \mathfrak{N}=$

$\therefore \dot{\sim} \dot{m}=$

$\div \div \div \div$

$0030=$

눙ㅇ․

$000<0$

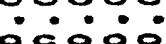

aㅁㅇㅇㅇ

in: 0

$\because \overrightarrow{1}: y$

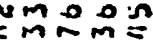

- N Nimn

บบบบ บ บ

20000

$\therefore \therefore \circ \circ \circ$

00000

$3=990$

$\overrightarrow{1} \overrightarrow{1} \overrightarrow{1}$

in $\operatorname{con}$

บmตทn

$0=00$

$\therefore \equiv \pm \approx=$

111\%

ñ $=0 \pi$

$\ddot{0} \dot{0} \dot{0}$

NNNN=

ำก

$\overrightarrow{\vec{n}} \overrightarrow{\mathrm{n}} \overrightarrow{\mathrm{n}}$

$\vec{c} \overrightarrow{0} \overrightarrow{0} 0$

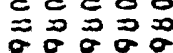

nONMn

$\therefore \dot{0}=\dot{m}$

뜬

프는

공잉ㅇㅇㅇ

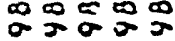

응ㅇㅇ웅

원의

$\overrightarrow{1} \div \overrightarrow{1}$

- ocro

ำง

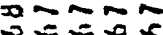

$\because \approx=0 \div$

on meo

$\rightarrow=5=m$

$\therefore \dot{0} \dot{0}$

00000

₹ $5=5$

$\ddot{1} \div \ddot{1}$

ติn

$m \sim 250$

-

$\leq 0 \leq \pm \leq$

$\alpha \alpha n \sigma$

mm的品

$\because \because \therefore \dot{0}$

-0000

ㄴ?

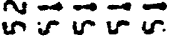

$\therefore 2000$

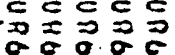

$n=0 \ddot{\sim}$

micó

등

50550

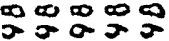

ЕैЕ ЕैЁ

Е ЕЕ E E

00000

00000

min

mmmm

웜의

$\dot{0} \div \dot{0}=$

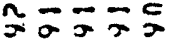

○ n

$n=0 x \infty$

$= \pm 0 m$

$\vec{a} \overrightarrow{0} \overrightarrow{0}$

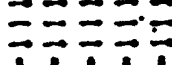

- 50

ธุบำ

$\therefore \dot{\pi}=$

수수

num

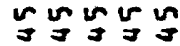

a nmo

으뭉

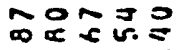

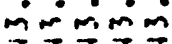

$\rightarrow=-0$

$\because= \pm=$

v. $\Rightarrow 5=0$

00.000

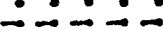

ruññ

nunm

]$\left._{3}\right]$

con $N$

กกับท

000

$1-20=$

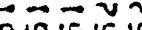

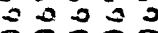

$\Rightarrow 200$

nคuno

oc $<$ ma

$\therefore \dot{0} \dot{0}$

203.

00030

$0 \%$

E E E E E

$0000=$

두웅

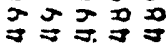

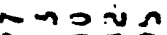




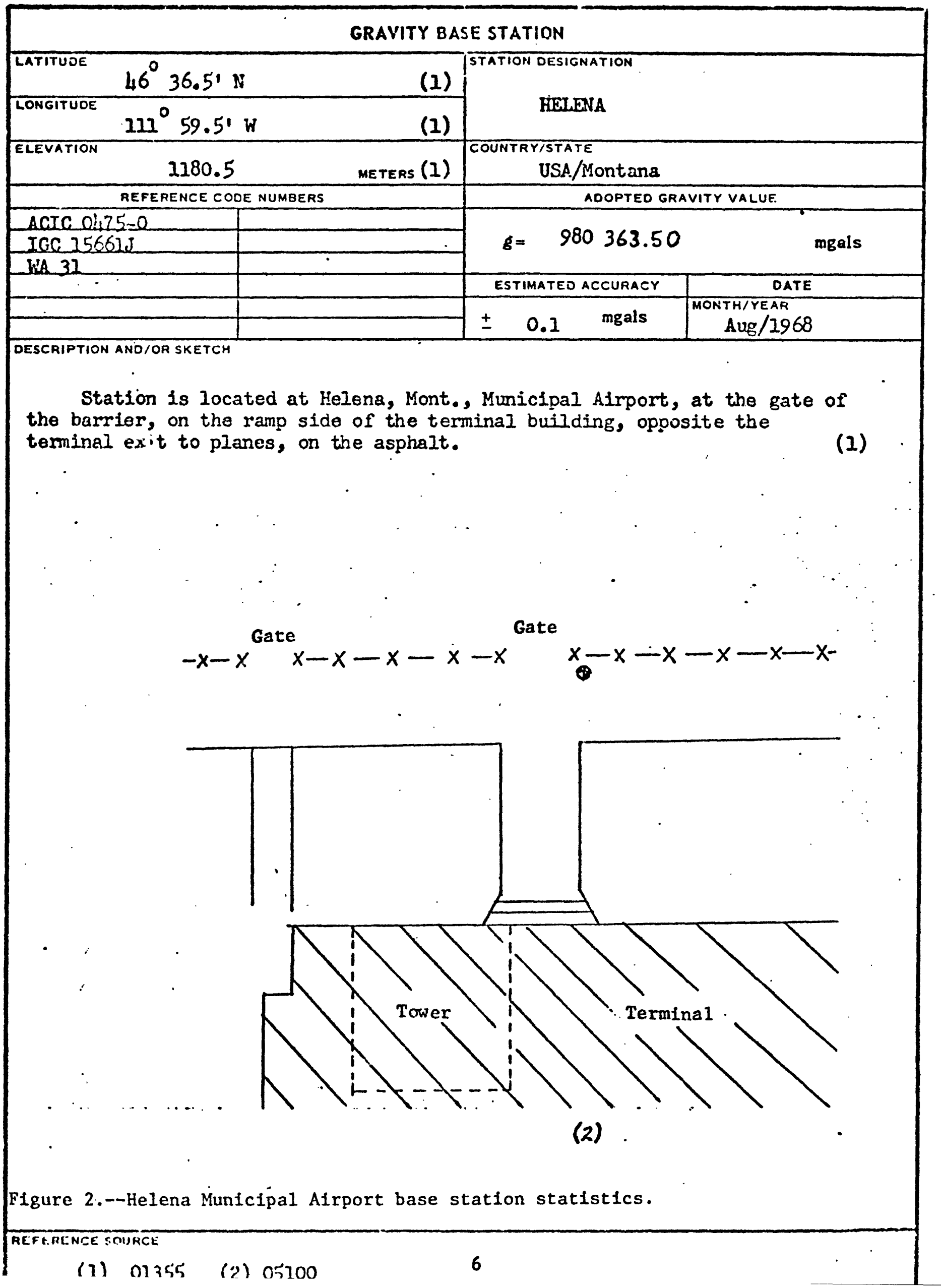




\section{References}

Defense Mapping Agency Aerospace Center, 1974, World Relative Gravity Reference Network, North America, Part 2, Defense Mapping Agency Aerospace Center Reference Publication 25, with supplement updating gravity values to the International Gravity Standardization Net 1971, 1635 p.

International Association of Geodesy, 1967, Geodetic Reference System, 1967: International Assoclation of Geodesy Special Publication 3, 74 p. 